\title{
Efeitos do reforço na pasta de cimento portland com óxido de grafeno obtido por métodos de química verde
}

\author{
Reinforcing effects on Portland cement \\ paste with graphene oxide obtained \\ Green chemistry methods
}

\author{
Milton Vizini Correa Neto ${ }^{1}$, Karynne Cristina de Souza ${ }^{1}$, \\ Fernanda Franco Massante ${ }^{2}$, Ananda Helena Heino Coelho ${ }^{2}$, \\ Eduardo Ariel Ponzio ${ }^{2}$, Jackson Antônio Lamounier Camargos Resende ${ }^{1}$
}

\begin{abstract}
${ }^{1}$ Programa de Pós-Graduação em Ciências de Materiais, Campus Universitário do Araguaia, Universidade Federal de Mato Grosso, Av. Valdon Varjão, N 6390, CEP: 78607-059. Barra do Garças, Mato Grosso, Brasil.

${ }^{2}$ Laboratório G2E, Departamento de Físico-Química, Campus Valonguinho, Universidade Federal Fluminense, Outeiro São João Batista, s/n CEP: 24.020-141. Niterói, Rio de Janeiro, Brasil.

e-mail: miltonvizini@gmail.com, karynnecsouza@yahoo.com.br,ffmcp2@gmail.com, anandaheino@id.uff.br, eduardoariel@id.uff.br, jacksonresende@ufmt.br
\end{abstract}

\section{RESUMO}

O objetivo do trabalho é sintetizar o óxido de grafeno (GO) de maneira mais sustentável e adicioná-lo à pasta de cimento Portland. O método de obtenção do GO mais comumente utilizado é o método de Hummers, em que a lavagem do material é feita com água, resultando em grande quantidade de rejeito aquoso ácido. Isso acarreta um problema de sustentabilidade ambiental desse processo, devido aos danos possíveis ao ambiente. Objetivando uma abordagem moderna nos preceitos da química verde para a obtenção do GO, propõe-se a substituição da lavagem com água pela neutralização da solução com carbonato de cálcio para a redução desses resíduos poluentes. Para comprovar a eficiência da síntese verde, fizeram-se comparações físicoquímicas nas amostras de GO obtidas, tanto pelo método Hummers (GO), quanto pelo método verde (GOv). As amostras foram analisadas por DRX, FTIR, RAMAN e MEV. As análises da suspensão de GOv não indicou a presença de novas fases cristalinas, porém observou-se a formação da fase gipsita na amostra seca na estufa. Os resultados mostraram a eficiência na produção de GO. Para a produção das pastas de cimento Portland foram feitos traços com a adição de 0,$1 ; 0,2 ; 0,3 ; 0,4 ; 0,5 ; 0,75$ e $1 \% \mathrm{~m} / \mathrm{m}$ de GOv e executados os ensaios de resistência à compressão dos corpos de prova. Foi observado um máximo de eficiência na propriedade de resistência mecânica com adição de 0,3\% de GOv no traço. Em estudos comparativos de microscopia eletrônica nos traços de $0,3 \%$ contra $1,0 \%$ de GOv pode-se constatar a agregação das lamelas de GO nos traços de maior concentração, o que resulta na menor resistência desses últimos. Assim, essa pesquisa apresentou uma proposta com menor impacto ambiental para a produção de compósitos de cimento com GO por um método simples.

Palavras-chave: Óxido de Grafeno. Cimento. Química verde.

\section{ABSTRACT}

The main objective of this paper is to synthesize graphene oxide (GO) in a more sustainable way and add it to Portland cement paste. The most commonly used method of obtaining GO is the Hummers method, in which the washing of the material is done with water, resulting in large amounts of aqueous acid waste. This process leads to environmental sustainability problem, due to the possible damages to the environment. Aiming for a modern approach in the precepts of green chemistry for obtaining GO, it is proposed to replace the washing with water by neutralizing the solution with calcium carbonate to reduce these toxic residues. To verify the efficiency of green synthesis, physicochemical comparisons of GO samples obtained by both the Hummers method (GO) and the green method (GOv) were performed. The samples were analyzed by XRD, FTIR and RAMAN, and SEM. The analyses of the GOv suspension did not indicate the presence of new crystalline phases, but the formation of the gypsum phase was observed in the dry sample in the hot chamber. The results showed the efficiency in the production of GO. For the production of Portland cement paste, we added 
a proportion of 0,$1 ; 0,2 ; 0,3 ; 0,4 ; 0,5 ; 0,75$ and $1 \%$ of $\mathrm{GOv}$ and, then compressive strength tests were performed on the specimens. A maximum efficiency in the mechanical strength property was observed with addition of $0.3 \%$ of GOv in the traces. In comparative studies of electron microscopy in the $0.3 \%$ traces against $1.0 \%$ of GOv traces, the aggregation of GO lamellae in the traces with higher concentration can be observed, which results in lower resistance of the latter. This research presented a proposal with less environmental impact for the production of cement composites GO by a simple method.

Keywords: Graphene Oxide. Cement. Green Chemistry.

\section{INTRODUÇÃO}

A produção brasileira de cimento chegou a 54 milhões de toneladas no ano de 2017 e atingiu o consumo per capita de 258,6 kg/hab/ano[1, 2]. Por ser um material amplamente utilizado na construção civil, pesquisas de novos compósitos com cimento para aumentar a resistência mecânica dos concretos se faz necessária, buscando a diminuição das dimensões das estruturas. A incorporação de nanomateriais ao cimento vem sendo realizada com esse intuito, visando alterar as propriedades macroscópicas dessas estruturas. Como exemplo, pode-se citar a aplicação dos nanotubos de carbonos para melhorar as propriedades da matriz cimentícia, incluindo resistência à flexão e tenacidade à fratura [3]. Os nanotubos de carbono oferecem melhoras substanciais nas propriedades mecânicas nos compósitos de cimento e concreto, bem como, promove a reatividade devido à sua grande área de superfície [4]. Outros tipos alótropos de carbono também podem ser utilizados como aditivos em cimentos, como por exemplo, o óxido de grafeno, sendo o grafite o material de partida para obtenção do mesmo.

Em geral, esses nanoaditivos, como GO, buscam aprimorar a hidratação do cimento Portland resultando num aperfeiçoamento de sua microestrutura. A inserção destes materiais, que interajam em níveis nano ou micro, leva a melhoria macroestrutural do cimento [5].

O grafite é um material barato, de uso imediato, disponível e precursor para os sistemas baseados em grafeno [5], sendo a principal forma alotrópica do carbono. Ele é descrito como um empilhamento de camadas de grafeno que interagem entre si através de forças intermoleculares de Van der Waals. O grafeno consiste em uma única camada desse átomo formando um sistema de anéis aromáticos. Ele é considerado um dos materiais mais resistentes e finos do mundo, com módulo de elasticidade da ordem de terapascal $[8,9]$. O interesse do óxido de grafeno (GO) advém das propriedades especiais que esse material possui, como sua elevada resistência mecânica, elevada área superficial e grupos funcionais ricos em oxigênio, que resultam em boa reatividade e boa dispersão em água do GO em relação ao grafeno [10].

O GO pode ser sintetizado pelos métodos de Brodie, Staudenmaier ou Hummers ou por uma variação do último, ou seja, o Método de Hummers modificado [11, 12, 13, 15]. Esses métodos são aplicados visando ao enfraquecimento das interações de Van der Waals do grafite e à introdução de grupos funcionais de oxigênio, promovendo a esfoliação e oxidação das folhas de grafeno em solução aquosa [15]. O método modificado de Hummers, em comparação aos demais métodos, resulta em um produto final com maior nível de oxidação e, normalmente, leva a um material com uma melhor performance [13]. Por exemplo, o GO, devido a sua grande área de superfície teórica, pode ser aplicado em processos de adsorção devido aos grupamentos funcionais contendo oxigênio. Uma outra propriedade importante é a facilidade de manipulação para gerar um material mais hidrofílico ou lipofílico, sendo a hidrofilicidade muito importante para facilitar a dispersão em soluções aquosas ou sob condições ambientais.

O GO tem emergido como um precursor de grafeno muito atraente para a produção em grande escala devido à capacidade de processamento em solução, estabilidade e facilidade de síntese. No entanto, o método modificado de Hummers, resulta na geração de gases NOx e resíduos ácidos resultantes da etapa de neutralização do material [13].

A Política Nacional de Resíduos Sólidos (PNRS) apresenta em seus princípios a prevenção e a precaução dos resíduos que são produzidos por uma indústria. Apesar dos resultados promissores para a aplicação de GO em diversos ramos da tecnologia, inclusive na indústria da construção civil, sua produção em larga escala leva a geração de um volume grande de resíduos [16, 17]. Nesse sentido, buscando uma forma de mitigar os resíduos ácidos, a pesquisa propõe apresentar em seu escopo uma alternativa na produção do GO, reduzindo seus resíduos e mantendo suas propriedades nos compósitos com cimento.

Vários estudos estão sendo desenvolvidos sobres efeitos do GO na durabilidade, nas propriedades mecânicas e reológicas $[4,17,18,19]$. Esse nanomaterial com grande área de superficial é um excelente sítio para reações de hidratação. No compósito de cimento com GO, esses grupos fazem interações fortes com moléculas de água, estabilizando sua absorção e gerando um reservatório de água e canais de transporte de água. Por esses motivos, o GO aumenta o teor de água não evaporável dentro da pasta de cimento e também 
induz o crescimento ordenado das fases de silicatos de cálcio hidratado $(\mathrm{CSH})$ e de portlandita $\left(\mathrm{Ca}(\mathrm{OH})_{2}\right)[4$, $20,21]$.

Devido aos grupos funcionais ligados, o GO tende a reagir com o CSH, a principal fase de ligação do cimento. Tal situação resulta na formação de uma interface quimicamente ligada entre o oxigênio (GO) e o cimento o que controla o crescimento das fases durante o envelhecimento da matriz cimentícia [5]. Como resultado, observa-se um cimento com resistência mecânica mais elevada. BABAK e colaboradores [5] descreveram que a adição de pequenas quantidades de GO ao cimento resultou em um aumento de até $44 \%$ de sua resistência mecânica.

Esse trabalho teve como objetivo produzir e caracterizar o GO obtido por um método ambientalmente sustentável e avaliar sua aplicabilidade em compósitos cimentícios, analisando o efeito da adição de GO na pasta de cimento sobre a resistência à compressão.

\section{MATERIAIS E MÉTODOS}

\subsection{Obtenção de GO}

Antes de iniciar a produção de GO, foi utilizada uma pré-esfoliação do grafite em acetona e água [22]. Nesse procedimento, colocou-se 0,500 g de Grafite (SYNTH G1013.06.AG) em uma solução composta de $45 \mathrm{~mL}$ de água deionizada e $135 \mathrm{~mL}$ de acetona (TEDIA). Essa suspensão foi colocada em um banho ultrassônico de $100 \mathrm{~W}$ por $4 \mathrm{~h}$. Em seguida, a acetona foi evaporada utilizando um rotaevaporador, e o material restante, contendo o grafite esfoliado, foi seco em estufa.

Para sintetizar o GO, foi utilizado o método de Hummers modificado: 0,500 g de grafite pré-esfoliado e $0,250 \mathrm{~g}$ de $\mathrm{NaNO}_{3}(\mathrm{SYNTH})$ foram transferidos para um balão de fundo redondo. Em seguida, adicionou-se $16,0 \mathrm{~mL}$ de $\mathrm{H}_{2} \mathrm{SO}_{4}(95,00 \%$ CRQ) em banho de gelo. A mistura foi deixada em agitação constante durante 1h. Após esse tempo, 1,500 g de $\mathrm{KMnO}_{4}(\mathrm{SYNTH})$ foi adicionado, gradualmente, controlando-se a temperatura para não exceder $20^{\circ} \mathrm{C}$. Após a completa dissolução do $\mathrm{KMnO}_{4}$, a mistura foi retirada do banho de gelo e elevou-se a temperatura para $30^{\circ} \mathrm{C}$, mantendo-se em agitação constante durante $12 \mathrm{~h}$. Após esse período, adicionou-se $250 \mathrm{~mL}$ de água deionizada e $3,0 \mathrm{~mL}$ de $\mathrm{H}_{2} \mathrm{O}_{2}$ (SYNTH) em agitação constante. Em seguida, o sistema foi deixado em repouso por mais $12 \mathrm{~h}$ para a sua decantação. Após esse período, o sobrenadante foi removido, restando $50 \mathrm{~mL}$ de uma suspensão ácida concentrada de GO.

Após essas etapas, foram efetuadas duas lavagens com solução de $\mathrm{HCl}(3 \%)$ para remover os metais restantes da solução. Esse procedimento foi repetido 9 vezes com o objetivo de produzir a quantidade de GO necessária para a preparação dos corpos de prova. Após essa etapa de lavagem com $\mathrm{HCl}$ e centrifugação, as suspensões ácidas de GO foram levadas a um balão volumétrico de $500 \mathrm{~mL}$ e o volume completado com água deionizada para o armazenamento (suspensão estoque inicial).

Ao final deste processo, a suspensão ácida contendo GO foi neutralizada. Com intuito de comparação química e física do GO, uma alíquota de $20 \%$ dessa suspensão foi separada para neutralização pelo método tradicional, utilizando-se apenas água deionizada e centrifugação. O restante da solução foi neutralizada com $\mathrm{CaCO}_{3}$, com objetivo de utilizar menor volume de água e esse produto foi utilizado também na preparação dos corpos de prova.

\subsection{Rota convencional de neutralização}

Nessa neutralização, o material foi lavado com água deionizada em várias etapas de centrifugação e remoção do sobrenadante ácido até completa neutralização do sobrenadante. Este processo resulta em um grande consumo de água, em média 3 litros de água por grama de óxido de grafeno.

\subsection{Rota verde para neutralização}

Com o intuito de reduzir a produção de rejeito aquoso ácido no processo de neutralização convencional, foi proposto um método mais sustentável de neutralização. Neste substitui-se as etapas de sucessivas lavagens com água pela adição de uma determinada quantidade de carbonato de cálcio. Para isso, a $80 \%$ da suspensão ácida contendo $\mathrm{GO}$ obtida no item 3.1 foi adicionada $\mathrm{CaCO}_{3}$ na proporção mássica de 1:1 de grafite inicial para $\mathrm{CaCO}_{3}$. Esse procedimento elevou o pH da suspensão para 7, gerando o GO denominado neste trabalho de óxido de grafeno verde (GOv). Então, foi realizado um ciclo de lavagem com centrifugação. Por fim, essa suspensão neutra de GOv foi diluída com água deionizada a um volume de $500 \mathrm{~mL}$ em balão volumétrico e armazenada (suspensão estoque de GOv).

O método de neutralização convencional foi, então, usado para comparação com a rota verde de neutralização utilizando-se das técnicas de espectroscopia vibracionais, difratometria e microscopia. Além disso, 
o método convencional foi usado para quantificar por gravimetria a concentração da suspensão produzida na etapa 3.1.

\subsection{Quantificação do Grafeno}

Para a quantificação de GO obtido no item 3.1, quatro alíquotas de $10,0 \mathrm{~mL}$ da suspensão estoque inicial foram retiradas utilizando-se uma pipeta volumétrica. Cada alíquota foi neutralizada por lavagem com água deionizada e centrifugação, seguindo o procedimento de neutralização convencional (item 3.2). Após a neutralização, a amostra foi quantitativamente transferida a um cadinho de porcelana e seca em estufa. O conteúdo de GO foi pesado. A massas obtidas são mostradas na Tabela 1.

Tabela 1: Massa de óxido de grafeno em 10,0 mL.

\begin{tabular}{c|c}
\hline AMOSTRA & MASSA DE GO (g) \\
\hline A & 0,122 \\
\hline B & 0,116 \\
\hline C & 0,117 \\
\hline D & 0,120 \\
\hline
\end{tabular}

Assim, a suspensão estoque inicial apresenta concentração média de $0,0119( \pm 0,0003) \mathrm{g} \mathrm{mL}^{-1}$ de GO.

\subsection{Preparação dos corpos de prova}

Os corpos de prova do compósito da pasta de cimento Portland e GOv foram preparados em moldes cilíndricos de PVC de 2,2 $\mathrm{mm}$ de diâmetro e 4,4 $\mathrm{mm}$ de altura, obedecendo a relação entre diâmetro e altura estabelecida pela NBR 7222 [23]. O cimento utilizado foi o Cimento Portland CPII-Z 32 da CIPLAN.

Para a confecção dos corpos de prova foi estimado o volume necessário a ser retirado da suspensão estoque neutralizada a fim de se obter a massa de GOv para os traços de 0,$10 ; 0,20 ; 0,30 ; 0,40 ; 0,50 ; 0,75$; $1,0 \%$ de GOv para $80 \mathrm{~g}$ de cimento e $40 \mathrm{~g}$ de água, em uma relação água/cimento $(\mathrm{a} / \mathrm{c})$ de 0,5 . Cada alíquota retirada da suspensão estoque foi transferida para uma proveta graduada de $50 \mathrm{~mL}$ e o volume foi completado para $40 \mathrm{~mL}$ utilizando água deionizada para a confecção dos corpos de prova (Tabela 2). No caso dos traços 0,75 e $1 \%$, os volumes retirados da suspensão estoque foram 63,2 e 84,2 mL, respectivamente. Esses excediam muito a relação água/cimento. Assim, as suspensões foram evaporadas em um béquer até o volume de $40 \mathrm{~mL}$.

Tabela 2: Traços utilizados para produção dos corpos de provas.

\begin{tabular}{c|c|c|c|c|c|c}
\hline $\begin{array}{c}\text { MASSA DE } \\
\text { CIMENTO } \\
(\mathbf{g})\end{array}$ & A/C & $\begin{array}{c}\text { ÁGUA } \\
(\mathbf{g})\end{array}$ & \% DE GOv & $\begin{array}{c}\text { MASSA DE } \\
\mathbf{G O}(\mathbf{g})\end{array}$ & $\begin{array}{c}\text { VOLUME DA } \\
\text { MISTURA (mL) }\end{array}$ & $\begin{array}{c}\text { ÁGUA } \\
\text { EVAPORADA }\end{array}$ \\
\hline 80 & 0,5 & 40 & 0 & 0 & 0 & - \\
\hline 80 & 0,5 & 40 & 0,10 & 0,080 & 8,4 & - \\
\hline 80 & 0,5 & 40 & 0,20 & 0,160 & 16,8 & - \\
\hline 80 & 0,5 & 40 & 0,30 & 0,240 & 25,3 & - \\
\hline 80 & 0,5 & 40 & 0,40 & 0,320 & 33,7 & - \\
\hline 80 & 0,5 & 40 & 0,50 & 0,400 & 42,1 & 23,2 \\
\hline 80 & 0,5 & 40 & 0,75 & 0,600 & 63,2 & 44,2 \\
\hline 80 & 0,5 & 40 & 1,0 & 0,800 & 84,2 & - \\
\hline
\end{tabular}

Após produção dos corpos de provas, eles foram deixados 28 dias curando em uma solução saturada de hidróxido de cálcio até serem realizados os ensaios de propriedade mecânica dos corpos de prova.

\subsection{Caracterizações}

As medidas de difração de raios $\mathrm{X}$ foram realizadas no difratômetro BRUKER (D8 ADVANCE) com um 
tubo com fonte de $\operatorname{Co~} \mathrm{K} \alpha(\lambda=1,79 \AA)$. As amostras foram analisadas no intervalo angular de $3^{\circ}$ até $70^{\circ} \mathrm{com}$ incremento de 0,02 .

Medidas de espectroscopia de absorção na região do infravermelho foram feitas em espectrofotômetro FTIR da Perkin-Elmer, modelo Spectometer 100, com resolução de $4 \mathrm{~cm}^{-1}$, na região compreendida entre 4000-600 $\mathrm{cm}^{-1}$. Os espectros foram obtidos à temperatura ambiente, utilizando-se acessório para a técnica de refletância total atenuada (ATR) com cristal de germânio $(\mathrm{Ge})$. As amostras foram secas e maceradas para serem analisadas em pó.

As medidas de espectroscopia Raman foram feitas no Microscópio confocal Raman Alpha 300, com software Witec e uma radiação incidente no comprimento de onda de $532 \mathrm{~nm}$, na faixa de varredura de 500 $\mathrm{cm}^{-1}$ e $2000 \mathrm{~cm}^{-1}$.

As imagens para o estudo da morfologia das amostras de GOv nos traços na proporção de 0,3\% e $1,0 \%$ GOv foram obtidas por microscopia eletrônica de varredura com elétrons secundários. Para isso, utilizou-se o microscópio eletrônico de varredura VEGA 3, da Tescan, operando com o feixe de elétrons sob tensão de aceleração de 30,0 kV. As imagens foram obtidas em ampliações de 3.000, 7.000, 14.000 e 17.000 vezes. Para obtenção das imagens, as amostras na forma de pó foram fixadas em fita de carbono em um porta amostra de alumínio.

Os ensaios de resistência à compressão foram feitos no equipamento de ensaios universais da TIME modelo WDW-50E. A velocidade de ensaio de $2 \mathrm{~mm} / \mathrm{min}$, sendo ensaiados 4 corpos de prova de cada condição testada.

Na Figura 1 é apresentado o esquema utilizado para preparação do GOv, partindo do grafite até a sua utilização final nos corpos de prova.

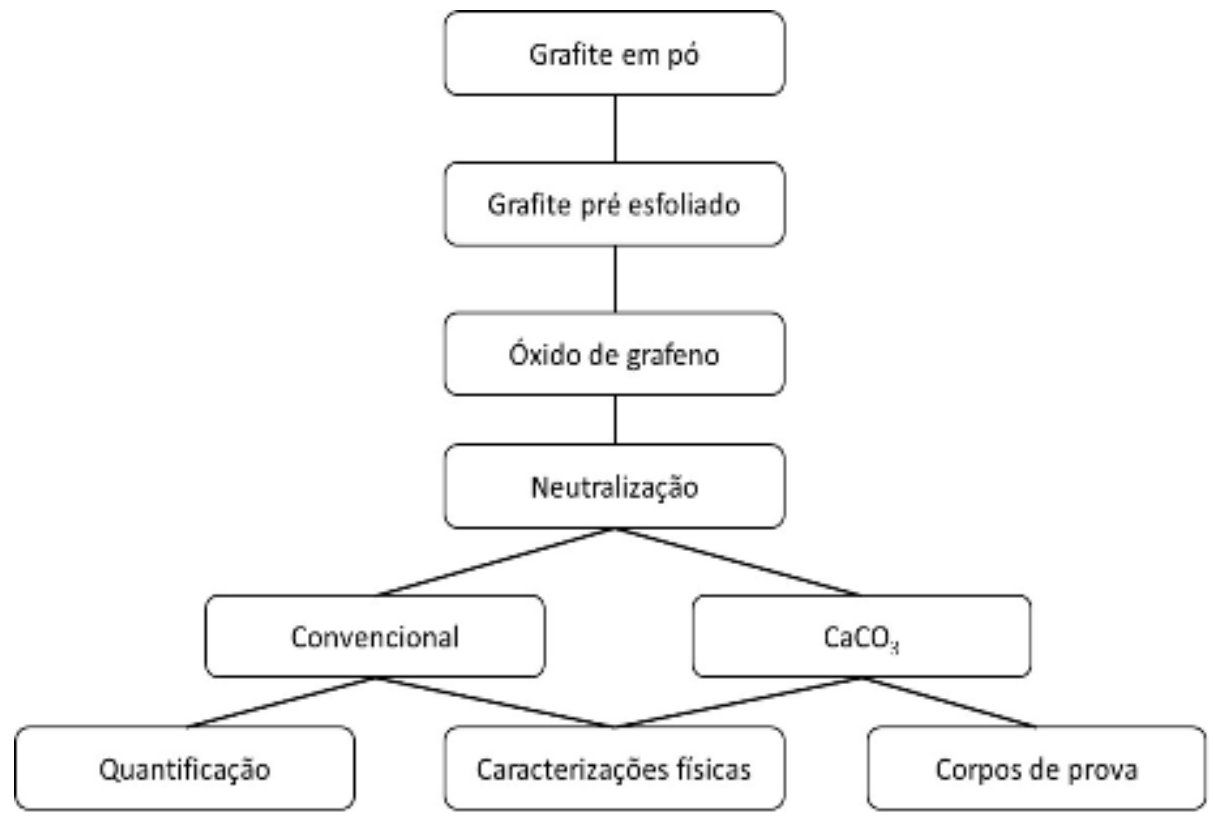

Figura 1: Esquema para produção dos corpos de prova.

\section{RESULTADOS E DISCUSSÔES}

\subsection{Comparação CO/GOv e Caracterizações Físicas e Químicas}

As amostras de GO e GOv foram caracterizadas técnicas de espectroscopia vibracionais (FTIR e RAMAN) difratométricas e por microscopia. Essas caracterizações visaram ilustrar a equivalência entre as espécies produzidas.

A Figura 2 ilustra os espectros de absorção na região do infravermelho das amostras GO e GOv. Observa-se as bandas em $3200 \mathrm{~cm}^{-1}$ e $1417 \mathrm{~cm}^{-1}$ que são atribuídas ao modo de vibração de deformação de grupos O-H. Uma banda em $1727 \mathrm{~cm}^{-1}$, característica do estiramento da carbonila $(\mathrm{C}=\mathrm{O})$ e $1606 \mathrm{~cm}^{-1}$, característica da ligação $\mathrm{C}=\mathrm{C}$. Foi observada a banda em 1090-1050 $\mathrm{cm}^{-1}$, associada à ligação C-O de grupos 
alcóxidos. Já a banda em $870 \mathrm{~cm}^{-1}$ pode ser atribuída aos grupos dos carbonatos $\mathrm{CO}_{3}^{-2}[8,14,25,26]$. Observa-se grande similaridade entre os espectros, e grande presença de água associada a banda larga entre $3200-3400 \mathrm{~cm}^{-1}$. Há poucas diferenças relevantes, sendo a principal o aumento na banda de $\mathrm{CO}_{3}{ }^{-2}$ atribuído a adição do carbonato de cálcio.

Figura 2: Espectro de absorção das bandas de Infravermelho do GO (vermelho) e do GOv (verde), indicando a grande similaridade entre as amostras e elevada presença de água associada a banda em $3200 \mathrm{~cm}^{-1}$.

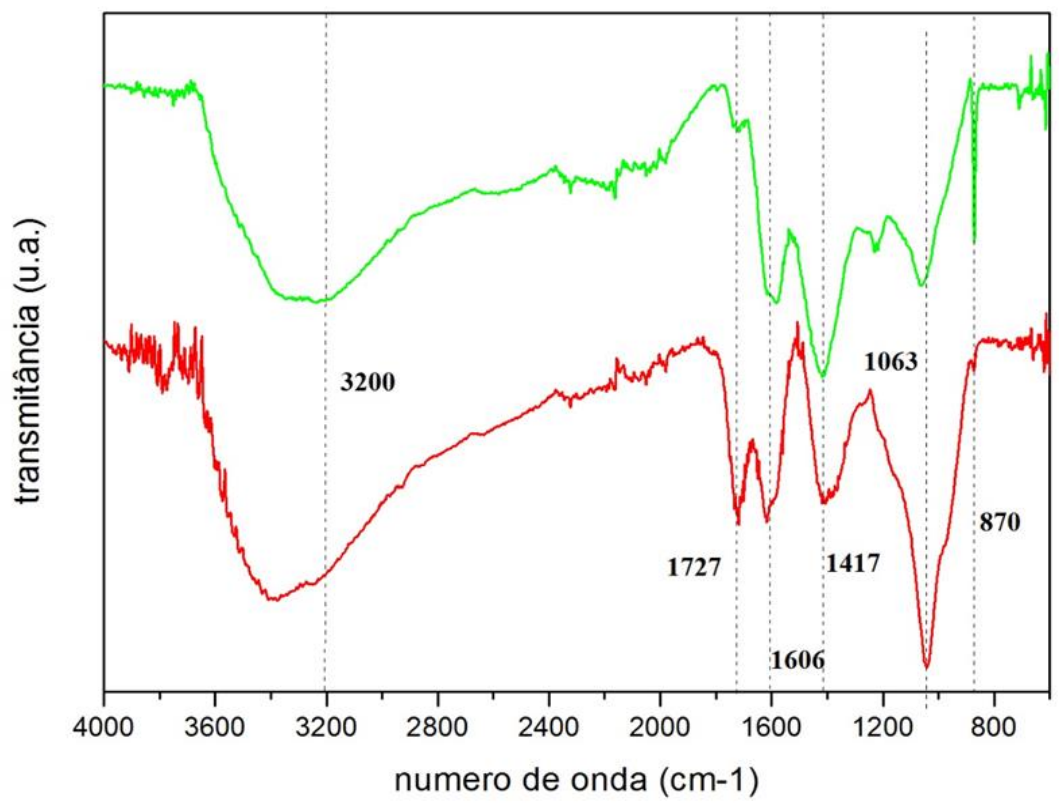

A Figura 3 ilustra os difratogramas de raios X (DRX) da amostra da suspensão GOv em comparação ao material seco na estufa a $80^{\circ} \mathrm{C}$. O padrão de difração do material produzido não apresenta picos de grafite, indicando eficiência do processo de síntese através da perda da estrutura cristalina [8, 27]. Observa-se um pico intenso de $\mathrm{GO}$ em $7,56^{\circ}$, valor típico para óxido de grafeno. O valor do espaçamento interplanar calculado $(1,36 \mathrm{~nm})$ é característico de sistemas com grande presença de grupos oxidados e de uma elevada retenção de moléculas de água na rede interlamelar do GO hidrofílico [13, 28, 29]. A amostra seca na estufa descreve a presença de gipsita $\left(\mathrm{CaSO}_{4} \cdot \mathrm{H}_{2} \mathrm{O}\right)$.

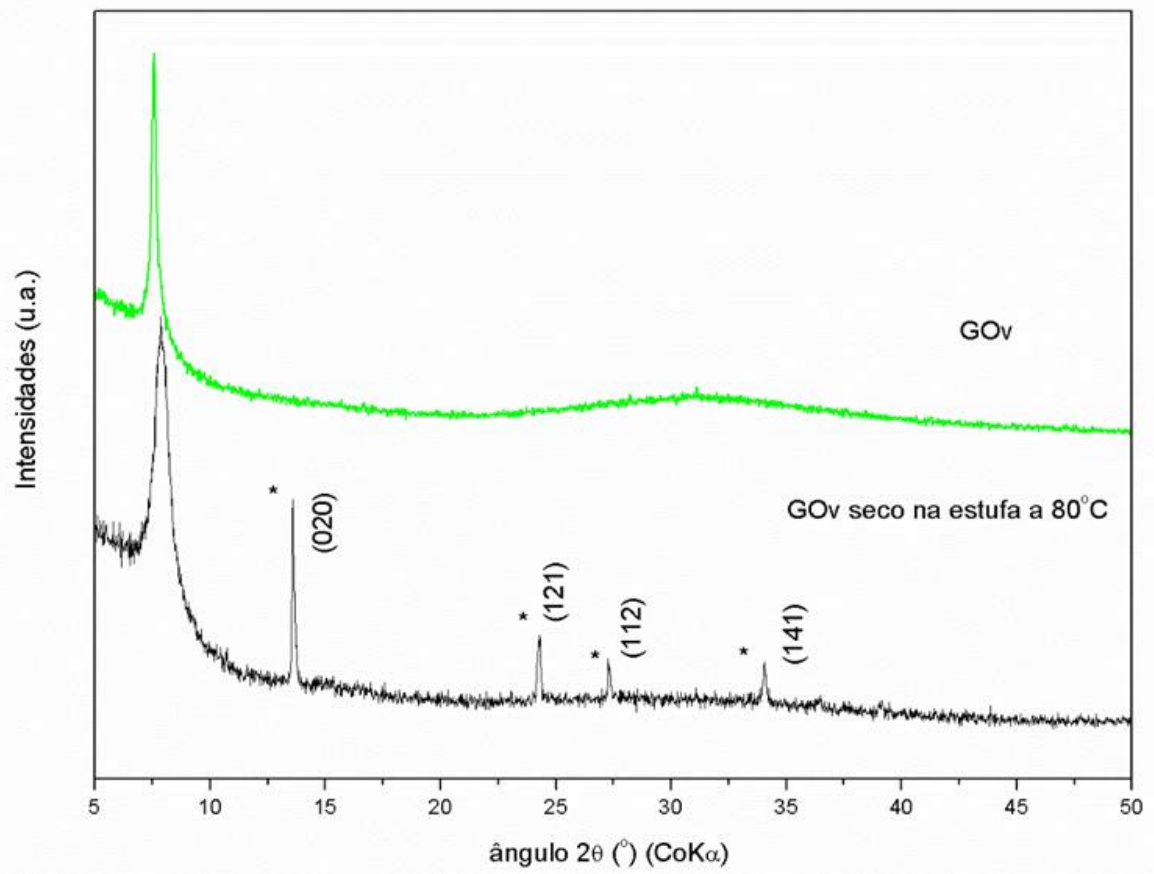


Figura 3: Comparação entre os padrões de difração de raios $X$ de policristais da suspensão de GOv (verde) com GOv seco na estufa (preto). * ilustra os picos de gipsita.

A espectroscopia Raman é uma ferramenta amplamente utilizada para a caracterização de produtos de carbono, especialmente considerando o fato de que ligações conjugadas e duplas de carbono levam a altas intensidades de Raman. Para caracterizar as duas amostras de óxido de grafeno foram obtidos os espectros Raman e estão apresentados na Figura 4.

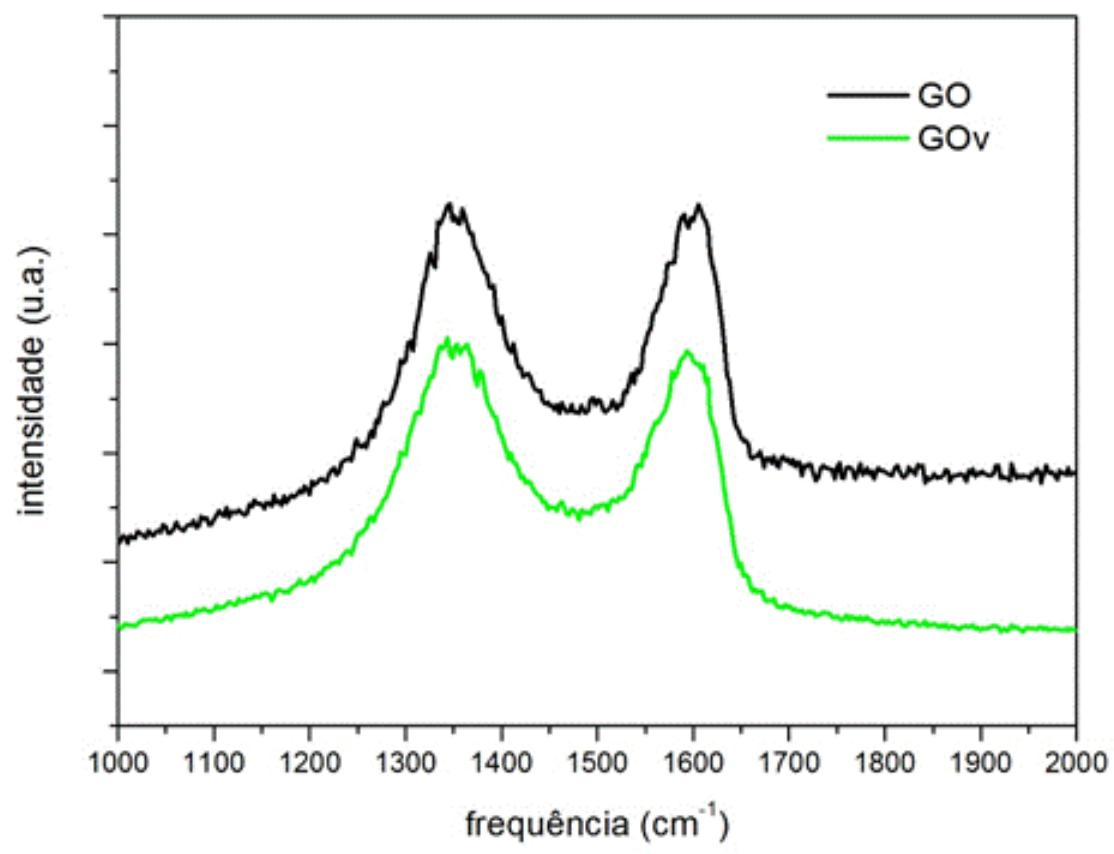

Figura 4: Comparação entre os espectros de Raman das amostras de óxido de grafeno, indicando suas similaridades.

Uma folha de grafeno pura exibe, tipicamente, uma banda em aproximadamente $1560 \mathrm{~cm}^{-1}$ (banda $\mathrm{G}$ ). A presença de banda adicional, próximo de $1350 \mathrm{~cm}^{-1}$ (banda D) é, geralmente, um resultado de defeitos $\mathrm{sp}^{3}$ na rede $\mathrm{sp}^{2}$, característica da formação de novas ligações decorrentes da oxidação dos átomos de carbono, típico do GO [30, 31]. Por isso, a razão de intensidade das bandas D e G (Id/Ig) fornece muitas informações sobre o grau de desordem na rede $\mathrm{sp}^{2}$; A relação de Id/Ig dos dois materiais é apresentada na Tabela 3 e está em acordo com o descrito na literatura [27, 32].

Tabela 3: Relação Id/Ig dos óxidos de grafeno.

\begin{tabular}{c|c}
\hline MATERIAL & Id/lg \\
\hline GO & 1,001 \\
\hline GOv & 1,002 \\
\hline
\end{tabular}

Com relação aos valores de Id/Ig obtidos, pode-se observar que os resultados da espectroscopia Raman das duas amostras descrevem materiais equivalentes. Isso indica que o grau de desordem dos dois materiais não apresentou nenhuma variação significativa. 
Nas imagens obtidas por MEV é possível observar as diferenças entre as morfologias apresentadas pelo grafite, grafite pré-esfoliado, GO e o GOv (Figura 5).
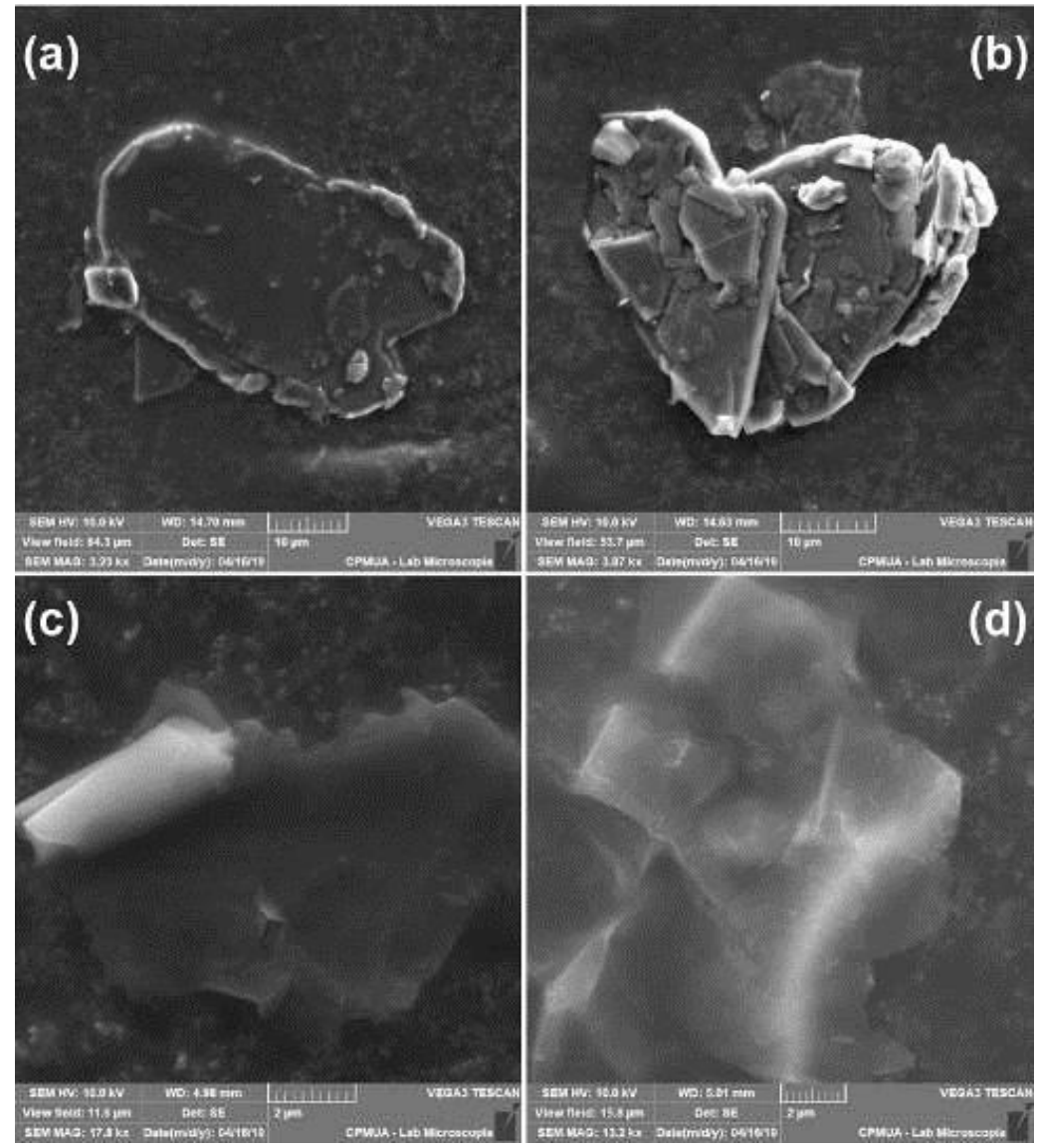

Figura 5: Micrografias das amostras obtidas pela técnica de MEV: Grafite (a), Grafite pré-esfoliado (b), GO (c), GOv (d).

Foi possível observar pela técnica de elétrons retroespalhados, a mudança da morfologia do grafite para o grafite pré-esfoliado, mostrando que o processo iniciou a esfoliação dos planos de grafite (Figura 5 (a) e (b)). Na Figura 5 (c) é possível ver uma folha de GO e na Figura 5 (d) pode-se observar o GOv que apresenta o mesmo padrão de folha do GO, indicando a delaminação da estrutura do grafite. Observa-se também a presença de uma fase diferente da esperada para o GOv: pequenos cristais, o que pode indicar a presença do sal de sulfato de cálcio monohidratado (gipsita) depositada na superfície do material (pontos mais claros da imagem). A presença de fase pode ser confirmada por difração de raios X na amostra seca em estufa (material suplementar).

Analisando os resultados obtidos pelas técnicas de caracterização, observou-se que o GOv possui as mesmas características apresentadas pelo GO. Desse modo, a utilização do GOv nos compósitos cimentícios no lugar do GO resultaria em uma redução dos resíduos, uma vez que sua neutralização é feita usando $\mathrm{CaCO}_{3}$ e não geraria o rejeito de água ácida resultante desse processo.

\subsection{Análise de resistência à compressão}

Uma análise visual, dos corpos de prova já rompidos no ensaio de resistência à compressão, é possível observar a mudança na coloração gradual nos corpos de prova. Tal fato indica que o material fez interação com a pasta de cimento e encontra-se bem disperso nos corpos de prova. 


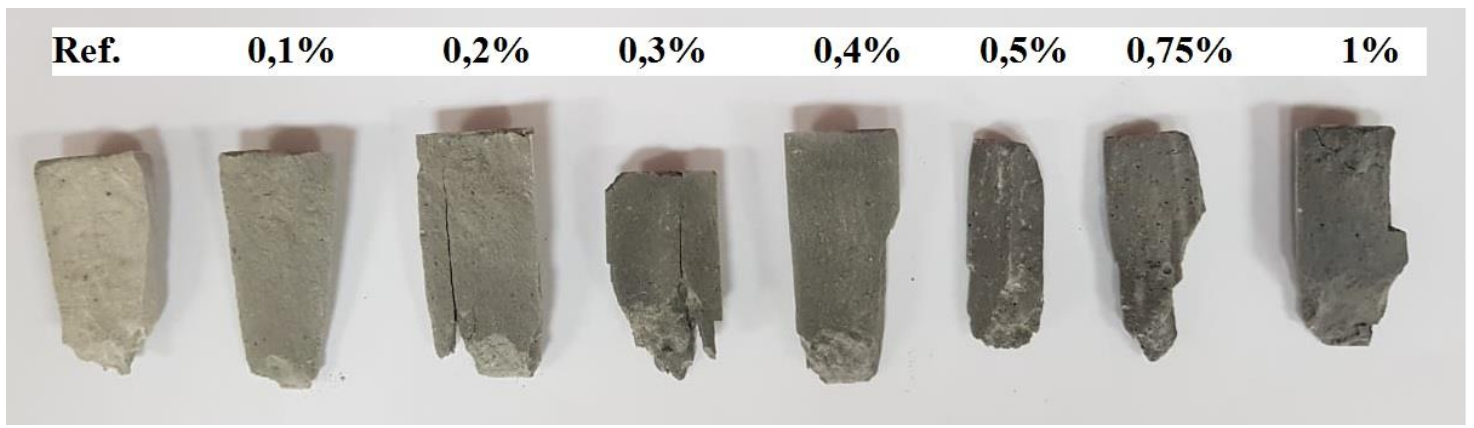

Figura 6: Fotografia interna do plano de fratura dos traços rompidos.

Os resultados obtidos de resistência à compressão dos corpos de prova de pasta de cimento Portland com o GOv são apresentados na Figura 7. Observou-se que para os corpos de prova de referência (sem adição de GOv) a resistência à compressão foi de $16,4( \pm 0,5) \mathrm{MPa}$. Quando foi adicionado $0,10 \%$ de GOv à pasta de cimento, observou-se um aumento de 14,7\% na resistência mecânica. Esse valor é similar ao descrito por KUDŽMA e colaboradores, que observaram o aumento de cerca de $15 \%$ ao adicionar a mesma quantidade de GO [35]. A série de ensaios mostrou um pico de resistência à compressão, quando foi adicionado $0,30 \%$ de GOv à pasta de cimento, com incremento de 65,6\% na resistência à compressão em relação ao material de referência. Os produtos de hidratação tendem a ser bem cristalizados com a adição de folhas de grafeno, pois favorecem o crescimento dos produtos de hidratação do cimento [31, 32]. Contudo, o aumento no teor de GOv em traços superiores a $0,30 \%$ resultou em redução da resistência à compressão da pasta de cimento em até 56,7\%, quando comparado com o valor de referência. Possivelmente, a causa desse efeito é a aglomeração de GOv nos capilares do cimento, dificultando sua interação com as fases do cimento, assim como foi observado por LIU, et al. [34] e BABAK, et.al. [5].

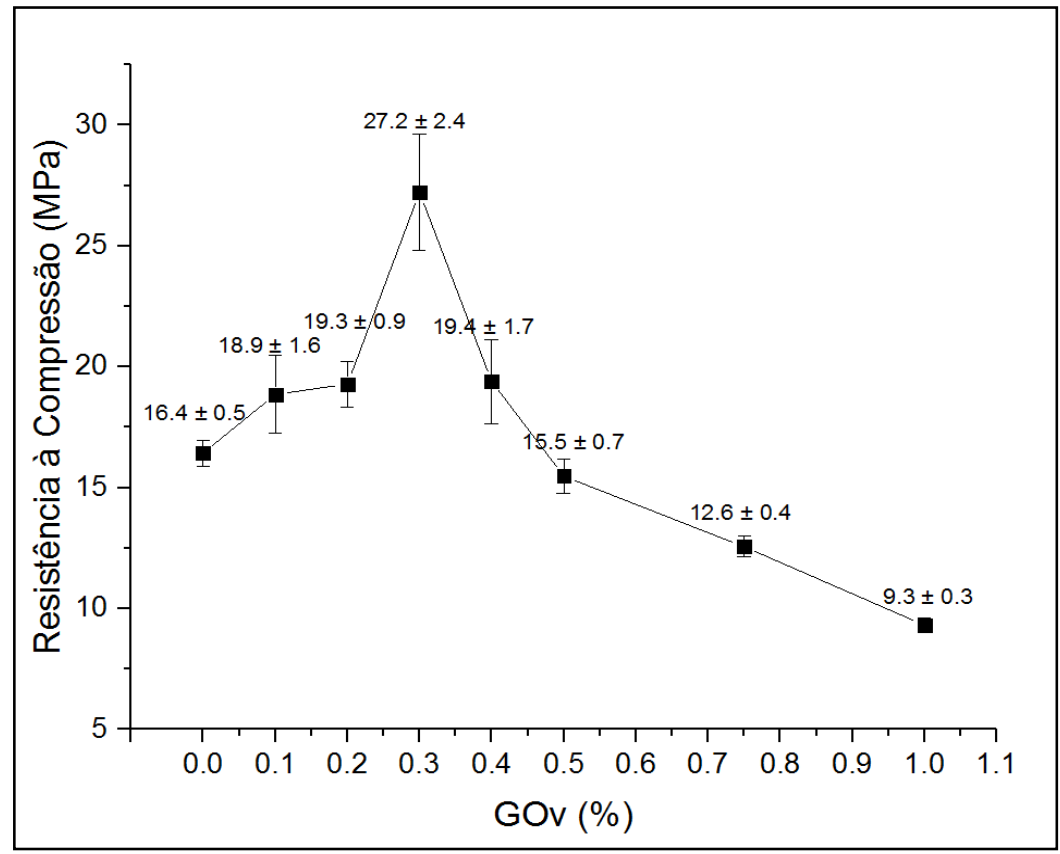

Figura 7: Descrição do comportamento da resistência à compressão média em relação a adição de GOv ao traço. 
BABAK e colaboradores [5], em pesquisas realizadas com GO e cimento, observaram que os produtos de silicatos de cálcio hidratado do cimento se depositaram na superfície GO, que atuou como um local de nucleação e os silicatos apresentaram uma grande adesão em sua superfície. KUDŽMA e colaboradores [35] observaram que a melhora nas propriedades mecânicas pode estar associada, principalmente, à compactação da estrutura, redução na descontinuidade e na porosidade da microestrutura da matriz cimentícia. Já WANG e colaboradores [20] observaram que dosagens de GO resultam em cristais de portlandita mais refinados. Na Figura 8 são ilustrados alguns resultados descritos na literatura, comparando o ganho percentual na resistência mecânica em relação ao corpo de prova de referência (sem adição de GO) dos trabalhos avaliados. Pode-se observar que o material produzido nesse artigo apresentou um ganho superior aos demais trabalhos comparados.

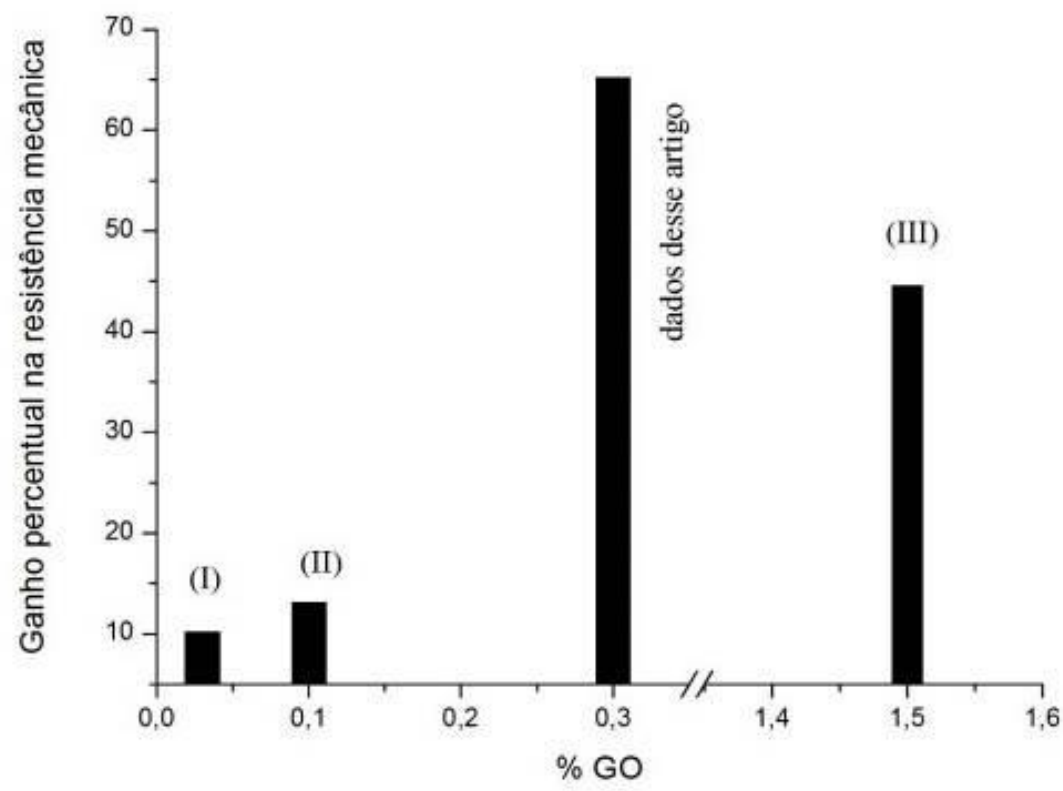

Figura 8: O ganho na resistência mecânica apresentado pelo grafeno e o GOv, onde (I) WANG, et al.[20], (II) KUDŽMA, et al.[35] e (III) BABAK, et al.[5]

A análise de microscopia (MEV) do corpo de prova com $0,30 \%$ e de 1,00\% de GOv é apresentado na Figura 9. Nessa figura podem ser observadas as folhas de GO, que atuam no sítio de hidratação, favorecendo o crescimento de cristais de fases portlandita e silicato de cálcio hidratado, como descrito na literatura [5,20,34]. Quando se adiciona até $0,30 \%$ do GOv na pasta de cimento, a nanopartícula apresenta boa dispersão na suspensão (baixa concentração), resultando em um nanomaterial melhor disperso na matriz cimentícia. Tal fato, levará à formação mais ordenada de cristais de portlandita e silicato de cálcio hidratado. Esse melhor ordenamento ocorre através dos planos de GO em interação com os íons de cálcio, que atuam como ponto inicial de crescimento dessas fases cristalinas. Em virtude disso, observou-se o melhor desempenho mecânico pela matriz [36]. Já nas adições superiores a 0,30\%, acredita-se que o as unidades de GO façam interação entre si formando agregados de GO, devido ao excesso da nanopartícula em suspensão. $\mathrm{Na}$ micrografia do corpo de prova com 1,0\% de GOv observa-se agregados de GO com tamanhos e números de camadas superiores ao do corpo de prova com $0,3 \%$ de GO. 


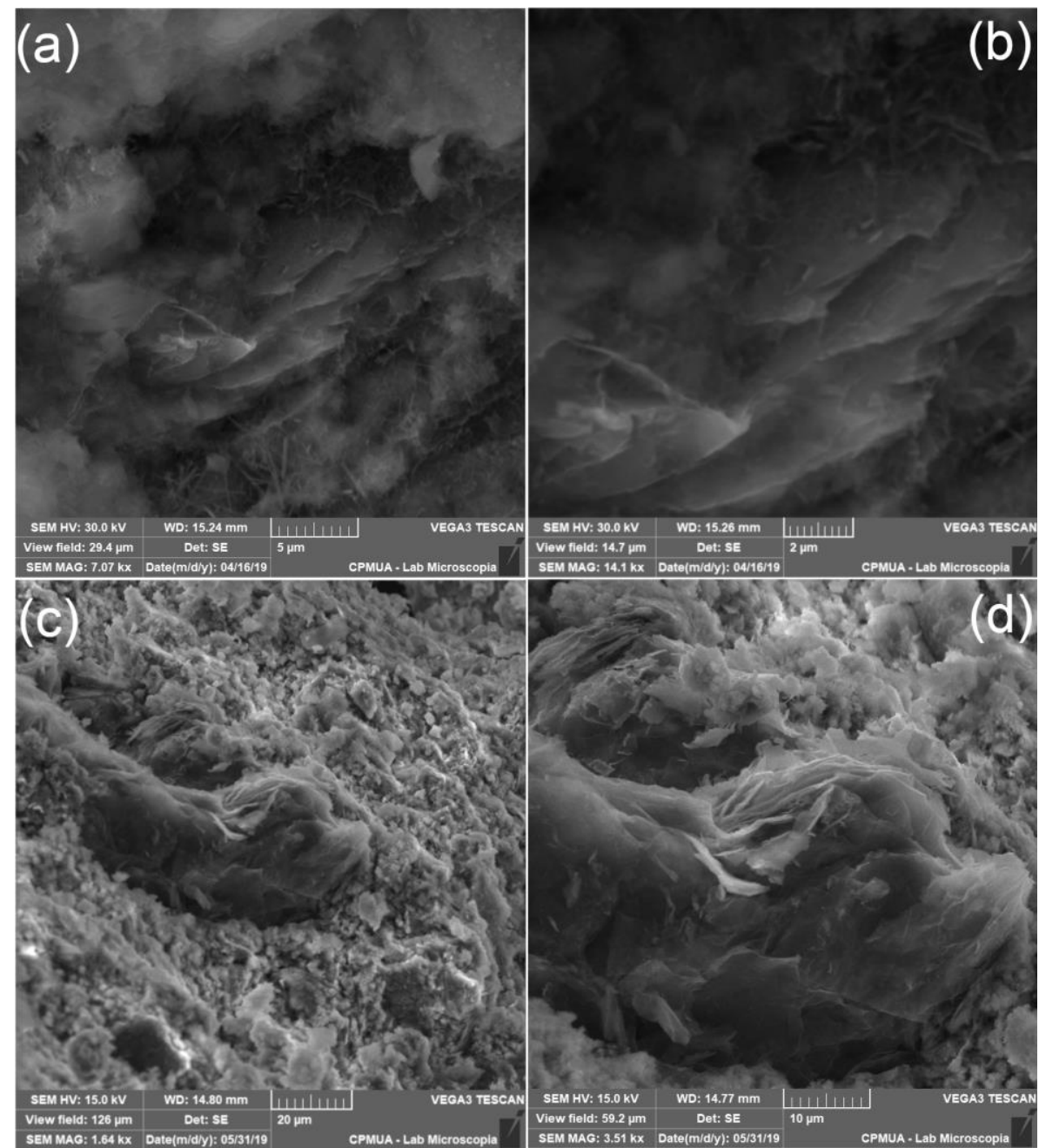

Figura 9: Micrografias dos traços obtidas pela técnica de MEV: 0,3\% de GOv (a) e (b), 1,0\% de GOv (c) e (d)

O difratograma de raios $\mathrm{X}$ dos corpos de prova de referência, de 0,3\% de GOv e de 1,0\% de GOv é apresentado na figura 10. Nestes foram possíveis constatar a presença das fases hidratadas do cimento Portland como a etringita, Portlandita, calcita e CSH. Em uma análise qualitativa das intensidades dos picos, pode-se observar que o traço de $1,0 \%$ apresenta os dois primeiros picos mais intenso que nos demais padrões. Esses picos são característicos da Etringita, indicando que no corpo de prova de 1,0\% há uma maior quantidade dessa fase. A presença da Etringita indica a possibilidade do aumento da fase CSH, em idade mais avançadas. 


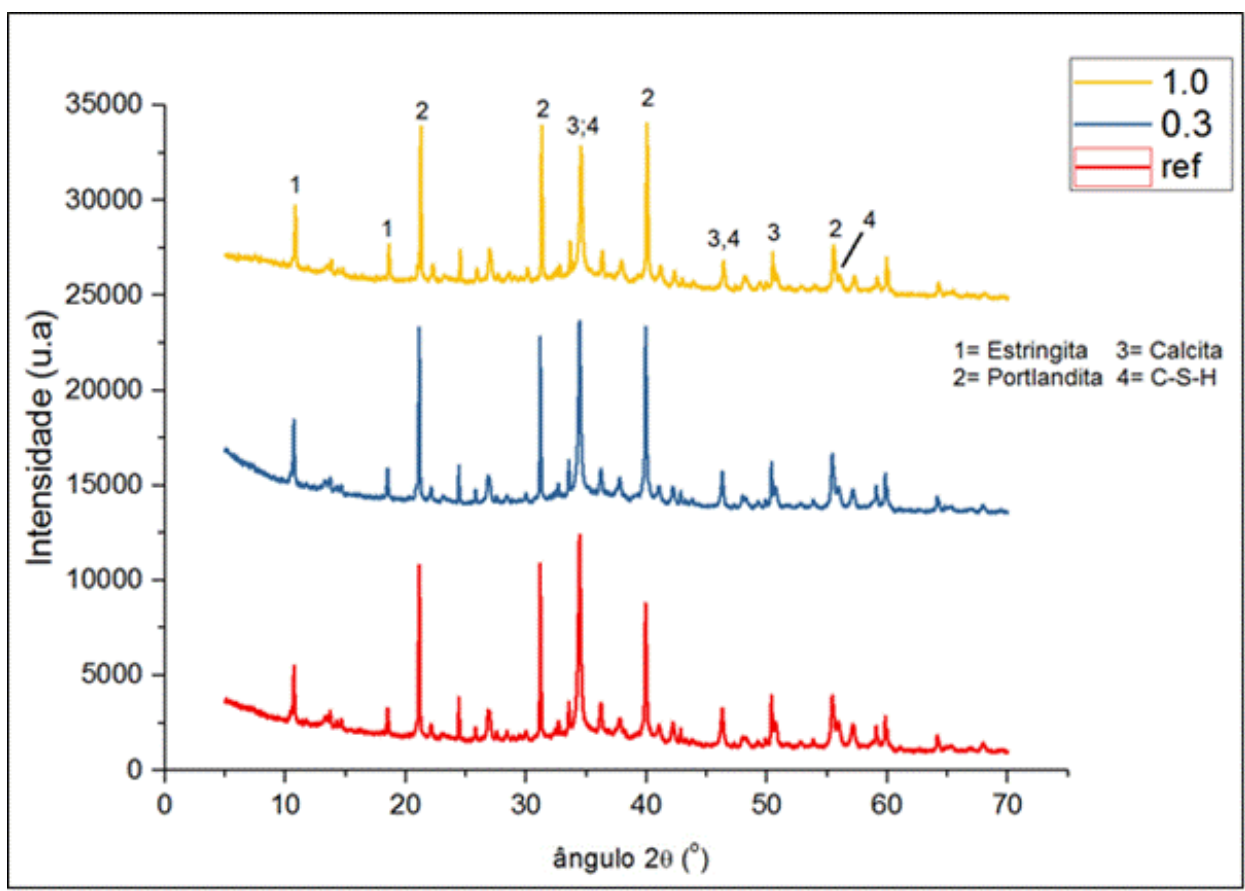

Figura 10: Difratogramas de raios $X$ dos corpos de prova de Referência (vermelho), 0,3\% (azul) e 1\% (amarelo).

Por fim, pode-se fazer uma propagação, sobre o traço de $0,30 \%$, do consumo de água necessária para preparação do GO em uma construção de interesse social, onde para cada $\mathrm{m}^{3}$ de construção utiliza-se, em média, 307,11 kg de cimento [37]. Assim, de acordo com os valores apresentados, seria necessário o uso de $922 \mathrm{~g} \mathrm{GO} / \mathrm{m}^{3}$. Para a produção dessa quantidade de GO, pelo método convencional, seriam consumidos de 3 mil litros de água adicionais por metro cúbico de construção. Esse volume pode ser economizado utilizandose o método proposto.

\section{CONCLUSÕES}

Pela modificação do método de Hummers na purificação do GO, foi possível apresentar uma rota sintética para o óxido de grafeno com produção de menor quantidade de resíduos, viabilizando-se, assim, um processo em acordo com os conceitos de química verde. A utilização do método proposto pode levar a um grande ganho ambiental e econômico com a grande economia de água nos processos produtivos.

Foi obtido óxido de grafeno com características equivalentes ao óxido de grafeno convencional e por um processo com potencialidade de aplicação em compósitos cimentícios. A aplicação do GOv na pasta de cimento Portland resultou no incremento da propriedade de resistência à compressão em até 65,6\%, com a adição de $0,30 \% \mathrm{~m} / \mathrm{m}$ de GOv em relação à massa de cimento. A associação desse ganho de propriedade mecânica com o método de síntese com menor consumo de água pode resultar em um grande ganho ambiental e econômico. Visto que se propõe um método com a grande economia de água nos processos produtivos e um nanocompósito cimentício com aumento de resistência mecânica para melhoria de estruturas.

\section{AGRADECIMENTOS}

O presente trabalho foi realizado com apoio da Coordenação de Aperfeiçoamento de Pessoal de Nível Superior - Brasil (CAPES) - Código de Financiamento 001 e CNPQ (grant 311142/2017-6; grant 313998/2017-5), Universidade Federal de Mato Grosso. Os autores agradecem ao Prof. Dante Franceschini, Laboratório de Filmes Finos do IF/UFF, pelas facilidades nas medidas RAMAN, e ao LDRX-UFF, pelas facilidades nas medidas de difração de raios $\mathrm{X}$.

\section{BIBLIOGRAFIA}

[1] SNIC, Sindicato Nacional da Indústria do Cimento. Produção nacional de cimento por regiões e estados. Rio de Janeiro: 2017.

[2] BRASIL. Estimativas populacionais para os municípios e para as Unidades da Federação brasileiros. Diário Oficial da União. Brasília, Jun.2017. 
[3] MAKAR, J. M.; MARGESON, J. C. ; LUH, J. "Carbon nanotube/cement composites - early results and potential applications". $3^{a}$ International Conference on Construction Materials: Performance, Innovation and Structural Implications: Vancouver, B.C. Ago. 2005.

[4] CHUAH, SAMUEL, et al. "Nano Reinforced Cement and Concrete Composites and New Perspective from Graphene Oxide". Construction and Building Materials, vol. 73, pp. 113-24, Dez. 2014.

[5] BABAK, FAKHIM, et al. "Preparation and Mechanical Properties of Graphene Oxide: Cement Nanocomposites". The Scientific World Journal, vol. 2014, pp. 1-10, Jan. 2014

[6] DUAN, Zhongcheng, et al. Experimental Test and Analytical Modeling of Mechanical Properties of Graphene-Oxide Cement Composites. Journal of Composite Materials, vol. 52, no 22, pp. 3027-3037, set. 2018

[7] ÇELIK, YASEMIN, et al. "A comparative study on few-layer graphene production by exfoliation of different starting materials in a low boiling point solvent". FlatChem, vol. 1, pp. 74-88, Jan. 2017.

[8] ROMERO, A., et al. Comparative Study of Different Scalable Routes to Synthesize Graphene Oxide and Reduced Graphene Oxide. Materials Chemistry and Physics, vol. 203, pp. 284-92, Jan. 2018.

[9] ZHU, YANWU, et al. "Graphene and Graphene Oxide: Synthesis, Properties, and Applications". Advanced Materials, vol. 22, nº 35, pp. 3906-24, Set. 2010.

[10] GU, XIANGYU, E JIAXIN PENG. "Preparation of NH2 Functionalized Graphene Oxide Nanosheets for Immobilization of NADH Oxidase". Journal of Nanoscience and Nanotechnology, vol. 18, $\mathrm{n}^{\circ}$ 6, pp. 3888-92, Jun. 2018.

[11] BRODIE, B. C. "XXIII.-Researches on the Atomic Weight of Graphite". Quarterly Journal of the Chemical Society of London, vol. 12, $\mathrm{n}^{\mathrm{o}}$ 1, pp. 261-68, Jan. 1860.

[12] HUMMERS, William S., Richard E. OFFEMAN. "Preparation of Graphitic Oxide". Journal of the American Chemical Society, vol. 80, nº 6, pp. 1339-1339, Mar. 1958.

[13] LAVIN-LOPEZ, MARIA DEL PRADO, et al. "Influence of Different Improved Hummers Method Modifications on the Characteristics of Graphite Oxide in Order to Make a More Easily Scalable Method". Industrial \& Engineering Chemistry Research, vol. 55, $\mathrm{n}^{\circ}$ 50, pp. 1-40, Dez. 2016.

[14] MARCANO, DANIELA C., et al. "Improved Synthesis of Graphene Oxide". ACS Nano, vol. 4, no 8, pp. 4806-14, Ago. de 2010.

[15] MUHAMMAD HAFIZ, SYED, et al. "A practical carbon dioxide gas sensor using room-temperature hydrogen plasma reduced graphene oxide". Sensors and Actuators B: Chemical, vol. 193, pp. 692-700, Mar. 2014.

[16] BRASIL. Lei $\mathrm{n}^{\circ}$ 12.305, de 2 de agosto de 2010. Institui a Política Nacional de Resíduos Sólidos; e dá outras providências. Diário Oficial da União. Brasília, 3 ago.2010.

[17] CHАBOT, V., et al. A review of graphene and graphene oxide sponge: material synthesis and applications to energy and the environment. Energy \& Environmental Science, v.7 , pp. 1564-1596.

[18] BIRENBOIM, Matan, et al. "Reinforcement and Workability Aspects of Graphene-Oxide-Reinforced Cement Nanocomposites". Composites Part B: Engineering, vol. 161, pp. 68-76, Mar. 2019.

[19] KRYSTEK, Malgorzata. "Mechanical properties of cement mortar with graphene oxide". Architecture Civil Engineering Environment, vol. 12, pp.91-96, Jan. 2019.

[20] WANG, QIN, et al. "Effect of Graphene Oxide on the Hydration and Microstructure of Fly Ash-Cement System". Construction and Building Materials, vol. 198, pp. 106-19, Fev. 2019.

[21] LIN, CHANGQING, et al. "Catalytic behavior of graphene oxide for cement hydration process". Journal of Physics and Chemistry of Solids, vol. 89, pp. 128-33, Fev. 2016.

[22] YANG, HAIBIN, et al. "Experimental study of the effects of graphene oxide on microstructure and properties of cement paste composite". Composites Part A: Applied Science and Manufacturing, vol. 102, pp. 263-72, Nov 2017.

[23] YI, MIN, et al. "Achieving concentrated graphene dispersions in water/acetone mixtures by the strategy of tailoring Hansen solubility parameters". Journal of Physics D: Applied Physics, vol. 46, n 2, pp. 025301, Jan de 2013.

[24] ASSOCIAÇÃO BRASILEIRA DE NORMA TÉCNICAS. NRB 7220 - Concreto e argamassa Determinação da resistência à tração por compressão diametral de corpos de prova cilíndricos. Rio de Janeiro, 2011. 
[25] EIGLER, SIEGFRIED, et al. "Formation and Decomposition of CO2 Intercalated Graphene Oxide". Chemistry of Materials, vol. 24, $\mathrm{n}^{\circ}$ 7, pp. 1276-82, Abr. 2012.

[26] MYNENI, SATISH C. B., et al. "Vibrational Spectroscopy of Functional Group Chemistry and Arsenate Coordination in Ettringite". Geochimica et Cosmochimica Acta, vol. 62, n. 21-22, p. 3499-514, Nov. 1998.

[27] STANKOVICH, SASHA, et al. "Synthesis of graphene-based nanosheets via chemical reduction of exfoliated graphite oxide". Carbon, vol. 45, nº 7, pp. 1558-65, Jun. de 2007.

[28] BLANTON, THOMAS N., E DEBASIS MAJUMDAR. "Characterization of X-Ray Irradiated Graphene Oxide Coatings Using X-Ray Diffraction, X-Ray Photoelectron Spectroscopy, and Atomic Force Microscopy". Powder Diffraction, vol. 28, nº 2, pp. 68-71, Jun. 2013.

[29] CAI, CHUJIANG, et al. "Facile and Size-Controllable Preparation of Graphene Oxide Nanosheets Using High Shear Method and Ultrasonic Method". Journal of Experimental Nanoscience, vol. 12, no 1, pp. 247-62, Jan. 2017.

[30] HACK, Renata et al. "Characterization of graphene nanosheets obtained by a modified Hummer's method”. Matéria (Rio J.), Rio de Janeiro, v. 23,n. 1, Mar. 2018

[31] GAYATHRI, S., JAYABAL, P., KOTTAISAMY M., RAMAKRISHNAN, V. Synthesis of Few Layer Graphene by Direct Exfoliation of Graphite and a Raman Spectroscopic Study. AIP Advances, vol. 4, no 2, pp. 027116, Fev. 2014.

[32] CHUA, CHUN KIANG, et al. "Graphite Oxides: Effects of Permanganate and Chlorate Oxidants on the Oxygen Composition". Chemistry - A European Journal, vol. 18, n 42, pp. 13453-59, Out. 2012.

[33] LV, SHENGHUA, et al. "Effect of Graphene Oxide Nanosheets of Microstructure and Mechanical Properties of Cement Composites". Construction and Building Materials, vol. 49, pp. 121-27, Dez. 2013.

[34] LIU, JINTAO, et al. "Fracture Toughness Improvement of Multi-Wall Carbon Nanotubes/Graphene Sheets Reinforced Cement Paste". Construction and Building Materials, vol. 200, pp. 530-38, Mar. 2019.

[35] KUDŽMA, ANDRIUS, et al. "Study on the Effect of Graphene Oxide with Low Oxygen Content on Portland Cement Based Composites". Materials, vol. 12, nº 5, pp. 802, Mar. 2019.

[36] LV, SHENGHUA, et al. "Structure, Performances, and Formation Mechanism of Cement Composites with Large-Scale Regular Microstructure by Distributing Uniformly Few-Layered Graphene Oxide in Cement Matrix". Structural Concrete, vol. 20, no 1, pp. 471-82, Fev. 2019.

[37] ASSUNÇÃO, J. W., Curvas de dosagem para concretos convencionais e aditivados confeccionados com materiais da região noroeste do Paraná. 2002. Dissertação (Mestrado) - UFSC, Florianópolis, 2002.

\section{ORCID}

Milton Vizini Correa Neto

Karynne Cristina de Souza

Fernanda Franco Massante

Ananda Helena Heino Coelho

Eduardo Ariel Ponzio

Jackson Antônio Lamounier Camargo Resende https://orcid.org/0000-0003-4398-2959

https://orcid.org/0000-0001-8508-2778

https://orcid.org/0000-0002-4829-6539

https://orcid.org/0000-0002-3483-2820

https://orcid.org/0000-0001-9630-8998

https://orcid.org/0000-0002-5366-4156 